\title{
Research Paper: Mental Arithmetic Task Recognition Using Effective Connectivity and Hierarchical Feature Selection From EEG Signals
}

\author{
Arash Maghsoudi ${ }^{1}$ (D), Ahmad Shalbaf ${ }^{2 *}$ (D) \\ 1. Department of Biomedical Engineering, Science and Research Branch, Islamic Azad University, Tehran, Iran. \\ 2. Department of Biomedical Engineering and Medical Physics, School of Medicine, Shahid Beheshti University of Medical Sciences, Tehran, Iran.
}

\begin{tabular}{|c|c|}
\hline $\begin{array}{l}\text { Use your device toscan } \\
\text { and read the article online }\end{array}$ & Citation Maghsoudi, A. \& Shalbaf, A. (2021). Mental Arithmetic Task Recognition Using Effective Connectivity and Hi- \\
\hline 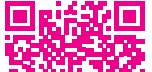 & $\begin{array}{l}\text { erarchical Feature Selection From EEG Signals. Basic and Clinical Neuroscience, 12(6), 817-826. http://dx.doi.org/10.32598/ } \\
\text { bcn.2021.2034.1 }\end{array}$ \\
\hline 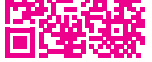 & doi http://dx.doi.org/10.32598/ben.2021.2034.1 \\
\hline
\end{tabular}

\section{(i) (3)}

Article info:

Received: 23 Sep 2019

First Revision: 19 Feb 2020

Accepted: 20 Apr 2020

Available Online: 01 Nov 2021

Keywords:

Electroencephalogram (EEG), Mental arithmetic, Effective connectivity, Feature selection

\begin{abstract}
A B S T RA C T
Introduction: Mental arithmetic analysis based on Electroencephalogram (EEG) signals can help understand disorders, such as attention-deficit hyperactivity, dyscalculia, or autism spectrum disorder where the difficulty in learning or understanding the arithmetic exists. Most mental arithmetic recognition systems rely on features of a single channel of EEG; however, the relationships between EEG channels in the form of effective brain connectivity analysis can contain valuable information. This study aims to find distinctive, effective brain connectivity features and create a hierarchical feature selection for effectively classifying mental arithmetic and baseline tasks.

Methods: We estimated effective connectivity using Directed Transfer Function (DTF), direct DTF (dDTF) and Generalized Partial Directed Coherence (GPDC) methods. These measures determine the causal relationship between different brain areas. A hierarchical feature subset selection method selects the most significant effective connectivity features. Initially, KruskalWallis test was performed. Consequently, five feature selection algorithms, namely, Support Vector Machine (SVM) method based on Recursive Feature Elimination, Fisher score, mutual information, minimum Redundancy Maximum Relevance (RMR), and concave minimization and SVM are used to select the best discriminative features. Finally, the SVM method was used for classification.

Results: The obtained results indicated that the best EEG classification performance in 29 participants and 60 trials is obtained using GPDC and feature selection via concave minimization method in Beta2 (15-22Hz) frequency band with $89 \%$ accuracy.
\end{abstract}

Conclusion: This new hierarchical automated system could be helpful in the discrimination of mental arithmetic and baseline tasks from EEG signals effectively.

\section{* Corresponding Author:}

Ahmad Shalbaf, PhD.

Address: Department of Biomedical Engineering and Medical Physics, School of Medicine, Shahid Beheshti University of Medical Sciences, Tehran, Iran. Tel: +98 (912) 2163201

E-mail: shalbaf@sbmu.ac.ir 


\section{Highlights}

- Propose effective connectivity to describe EEG signals during mental arithmetic task.

- Most significant connectivity features from generalized partial directed coherence method.

- Hierarchical feature selection from Kruskal-Wallis test and concave minimization method.

\section{Plain Language Summary}

Brain analysis methods by Electroencephalogram (EEG) signals provide a suitable method to monitor human brain activity due to having high temporal resolution, being noninvasive, inexpensive, and portable method. Analysis of mental arithmetic based EEG signal is helpful for psychological disorders like dyscalculia where they have learning understanding arithmetic, attention deficit hyperactivity, and autism spectrum disorders with attention deficit problem. This study finds distinctive effective brain connectivity features and creates a hierarchical feature selection for classification of mental arithmetic and baseline tasks effectively. Best EEG classification performance in 29 participants and 60 trials is obtained using Generalized Partial Directed Coherence (GPDC) methods and feature selection via concave minimization method in Beta2 (15-22Hz) frequency band with $89 \%$ accuracy. Thus, this new hierarchical automated system is useful for discrimination of mental arithmetic and baseline tasks from EEG signal effectively.

\section{Introduction}

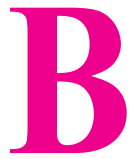

rain analysis methods provide a suitable process to monitor human brain activity (McFarland \& Wolpaw, 2008; Wolpaw, Birbaumer, McFarland, Pfurtscheller, \& Vaughan, 2002). For practical applications, Electroencephalogram (EEG) is very convenient due to having a high temporal resolution being a noninvasive, inexpensive, and portable method. EEG devices could be set up within minutes and used efficiently in real-time clinical applications (Shalbaf, Shalbaf, Saffar, \& Sleigh, 2020; Mohseni, Maghsoudi, \& Shamsollahi, 2006; Afshani, Shalbaf, Shalbaf, \& Sleigh, 2019; Neghabi, Marateb, \& Mahnam, 2019; Akbarian, \& Erfanian, 2018).

Analysis of mental arithmetic-based EEG signal is helpful for psychological disorders like dyscalculia where they have learning understanding arithmetic (Tajar \& Sharifi, 2011), attention-deficit hyperactivity (Lubar, Swartwood, Swartwood, \& O'Donnell, 1995), and autism spectrum disorders with the attention-deficit problem (Thompson, Thompson, \& Reid, 2010). In the past, several signal processing techniques from singlechannel EEG were proposed to separate mental arithmetic tasks from the baseline state, namely, calculation of the autoregressive model coefficients (Liang, Saratchandran, Huang, \& Sundararajan, 2006), power spectral density using Fourier transform at useful frequency bands (Harmony et al., 1999; Rebsamen, Kwok, \& Pen- ney, 2011), and generalized Higuchi fractal dimension spectrum (Wang \& Sourina, 2013). However, none of these methods have been proved to be adequately reliable in practical settings because of using the one-channel EEG features and ignoring valuable information inherent between multi-channels of EEG signals. Analyzing a single channel can hardly achieve reasonable accuracy. Mental arithmetic task causes complicated neurophysiological changes, and consequently, the relationships among brain regions are essential for solving the limitation mentioned above and providing more valuable features.

Nowadays, a new concept in brain integration focuses on brain connectivity. The broad field of brain connectivity analysis has two general subdivisions (Lang, Tome, Keck, Gorriz, \& Puntonet, 2012): functional connectivity and effective connectivity. Numerous functional connectivity methods such as Pearson correlation and coherence have been proposed to evaluate the relationship between simultaneous signals during mental arithmetic task recognition (Dimitrakopoulos et al., 2017; Dimitriadis, Sun, Kwok, Laskaris, \& Bezerianos, 2013). However, these techniques are only based on changes in statistical correlations between indirect observations of neuronal activities. Thus, characterizing brain dynamics using effective connectivity, which represents the causal interactions among brain regions, can be a valuable tool and has received much attention in recent years in the study of the brain (Seth, 2010). A study employed delay symbolic transfer entropy as an effective connectivity 
method with relative success in mental arithmetic recognition (Dimitriadis, Sun, Thakor, \& Bezerianos, 2016). Consequently, this study aims to find distinctive, compelling connectivity features and develop a hierarchical feature selection and classification method to discriminate mental arithmetic vs. resting state.

\section{Methods}

In this study, twenty-nine healthy individuals were without reported neurological, psychiatric, or other brainrelated diseases (15 females \& 14 males, Mean \pm SD age: $28.5 \pm 3.7$ years) (Shin et al., 2017). According to the Ethics Committee of Technical University of Berlin (SH_01_20150330).

Thirty electrodes of EEG data were recorded at 1000 $\mathrm{Hz}$ sampling rate by BrainAmp EEG amplifier according to the international 10-5 system with Fz as the reference electrode. The research participants sat at a $1.6 \mathrm{~m}$ distance from a 50-inch white screen with nobody moving. Three sessions of the experiment were performed on mental arithmetic and baseline task. Each session included a 1 min resting in pre and post-experiment and 20 repetitions (10 trials for each mental arithmetic \& baseline task). The task period is $10 \mathrm{~s}$ and 15 to $17 \mathrm{~s}$ resting period randomly. Figure 1 shows the schematic diagram of the experimental paradigm. In the mental arithmetic task period, participants repeatedly perform a subtraction like $384-8$, and in the baseline task, no specific action is done. The mental arithmetic and baseline tasks were randomized in $10 \mathrm{~s}$ of a task period (Shin et al., 2017). Therefore, for each participant in the three sessions, 30 trials for the mental arithmetic task and 30 trials for the baseline task in $10 \mathrm{~s}$ of a task period were performed.

Effective connectivity describes causal interactions and representations of the direction and power of the information flow among different brain areas (Astolfi et al., 2007). Granger Causality (GC) is a popular statistical method to estimate effective connectivity is Granger Causality (GC), i.e., based on a data-driven approach (Granger, 1969; Geweke, 1984). This method is based on estimating parameters of the Multi-Variable Auto-Regressive (MVAR) model for individual signal data. Quantitative spectral GC measures are: Generalized Partial Directed Coherence (GPDC) (Baccalá, \& Sameshima, 2001; Baccalá, Sameshima, \& Takahashi, 2007), Directed Transfer Function (DTF) (Korzeniewska, Mańczak, Kamiński, Blinowska, \& Kasicki, 2003), and direct DTF (dDTF) (Kaminski, Ding, Truccolo, \& Bressler, 2001). These techniques determine directional influences among different EEG channels per specific frequency range. We extract each frequency range for each measure by averaging theta $(4-7 \mathrm{~Hz})$, alpha or $\mathrm{mu}(8-12 \mathrm{~Hz})$, beta1 $(12-15 \mathrm{~Hz})$, beta $2(15-22 \mathrm{~Hz})$, beta $(22-30 \mathrm{~Hz})$, and gamma $(30-45 \mathrm{~Hz})$ frequency bands (the results of lowfrequency delta band was not reported here due to low accuracy classification). All calculations were done in MATLAB (The Mathworks, Inc., Natick, MA, USA) via the open-source SIFT toolbox (Mullen, 2010).

Due to the limited dataset, k-fold cross-validation was used. In our work, through trial and error, we used various amounts of $\mathrm{K}$. We found out that $\mathrm{K}=10$ yields the best results in terms of maximizing the classification performance and minimizing overfitting. In each trial, the classification structure is constructed with $90 \%$ of data $(80 \%$ for the training \& $10 \%$ for validation) and evaluated with the remaining data as testing data. The procedure is repeated 10 times, with each subsample as the testing data until all the dataset has been used for testing, and evaluation performance is reported by averaging the 10 results. Moreover, the 10-fold cross-validation is done for 100 consecutive runs, and the average of the results is calculated.

The EEG data were re-referenced using a typical average reference, and the fourth-order of Chebyshev passband filter of 2-45 Hz was done. Moreover, EOG rejection is done using independent component analysis in EEGLAB (Delorme \& Makeig, 2004).

The statistical significance of the extracted features obtained from effective connectivity methods between mental arithmetic tasks vs. resting states is studied by the Kruskal-Wallis test (Spurrier, 2003). Insignificant features with $\mathrm{P}>0.01$ were deleted. Next, 5 widely featured selection algorithms choose the best parts. The first method is Recursive Feature Elimination based on Support Vector Machine (SVM-RFE) (Guyon, Weston, Barnhill, \& Vapnik, 2002). This method uses SVM to score a given subset of features and selects features in a sequential, backward elimination manner. The other three feature selection methods are the Fisher score $(\mathrm{Gu}$, Li, \& Han, 2011). Mutual Information (MI) (Zaffalon, $\&$ Hutter, 2002), and minimum Redundancy Maximum Relevance (mRMR) (Ding, \& Peng, 2005; Peng, Long, \& Ding, 2005). In these three approaches, the selection of features is based on the general characteristics of data and ignores the classifier. Attributes are evaluated, and a subset is extracted. Fisher's method calculates a score as the ratio of interclass separation and intra-class variance for a feature. The MI method considers the MI between the distribution of the feature values and the membership to a specific class. Then, the final feature selection in these two methods is made by collecting top rankings. 
In the mRMR algorithm, features can be selected to be mutually far away from each other while still having the highest relevance to the target classes. Eventually, we have used feature selection via concave minimization and SVM (Bradley \& Mangasarian, 1998). In this method, the feature selection procedure is injected into the training of the SVM classifier, and the learning and the feature selection parts cannot be separated.

This binary classifier is used to categorize the data that the margin between the hyperplane and nearest data becomes maximum (Bradley \& Mangasarian, 1998). In this method, when overlapped features exist, Support vector classification maps the component into a higher dimension space by quadratic, polynomial, or Radial Basis Function (RBF). It creates a good discriminatory hyperplane in that space.

\section{Results}

Spectral effective connectivity measures (GPDC, DTF, dDTF) based on the calculated MVAR model parameters were estimated based on each signal frequency band range (theta, alpha, beta1, beta2, beta3, and gamma). All trials of each subject's session were considered an observation for classification $(3 \times 29$ observations for each class $)$. In each session, 10 trial runs for each task (10 seconds of data with frequency sampling $=1000 \mathrm{~Hz}$ ), leading to 100000 samples, are considered. Thus, we fit one MVAR model with the order of 60 to each observation with 100000-time samples. These 100000-time samples and order of 60 are selected according to the whiteness of residuals, stability, and consistency of the model (using built-in SIFT toolbox procedure called pop_est_validateMVAR).

Having 30-channel EEG, $900(30 \times 30)$ directed causal connection between channels as effective connectivity features are extracted for each GC method in each frequency band range that makes further computations complex. Accordingly, we performed a feature selection method based on the Kruskal-Wallis statistical test for each feature vector element, comparing the data of the two interest groups to choose the most significant features for discrimination of mental arithmetic task and baseline state. Using this test, the nonsignificant features with $p>0.01$ are deleted. Then, using the five feature selection algorithms (SVM-RFE, Fisher, MI, mRMR, and feature selection via concave minimization and SVM), the best features remained after the Kruskal-Wallis test are selected. Finally, the best-selected features are fed to the SVM classification structure to classify EEG data into mental arithmetic tasks accurately vs. resting state in 29 participants. A 10-fold cross-validation was used. In our case, we used $10 \%$ of the data for test and $90 \%$ for training and validation ( $80 \%$ train and $10 \%$ validation). In our methodology, through trial and error, we evaluated different kernels and different parameters in validation data and finally used an SVM with RBF kernel and sigma of 0.9 . The diagram of the proposed method is demonstrated in Figure 2.

The classification accuracies obtained by the spectral effective connectivity measures (GPDC, DTF, dDTF) and 5 widely featured selection methods over all participants for each frequency band range are given in Table 1, separately. Accordingly, GPDC and feature selection via concave minimization yields the best results with high accuracy within beta2 (89.05) frequency bands of EEG. Raw $900(30 \times 30)$ connectivity features for the GPDC method for Beta2 frequency band over all participants for mental arithmetic task vs. resting state are shown in Figure 3. Moreover, the best selected of GPDC connectivity features obtained from feature selection via concave minimization and SVN for Beta2 frequency band over all participants for mental arithmetic task vs. resting state are shown in Figure 4. In these two Figureures, a higher absolute value of connectivity feature is presented using warmer colors. A higher absolute difference value of connectivity in Figure 4 between mental arithmetic task vs. resting state means a better separability with higher significance. Furthermore, during the mental arithmetic task, EEG generally indicated high separability around the frontal and prefrontal areas (Figure 4).

\section{Discussion}

A new automated method for discriminating mental arithmetic tasks vs. resting state from EEG signal in 29 participants. We used the effective pairwise connectivity among different brain regions in different frequency bands as features in the hierarchical machine learning classification algorithm. Satisfactory classification accuracy was obtained. One of our novelties is using the GPDC method and also compareing it with other methods of effective connectivity such as DTF and dDTF in multi-frequency bands. Effective connectivity values using GPDC could effectively quantify the EEG signals' complexity during a mental task. Another novelty of our paper is proposing a hierarchical machine learning structure based on two feature selection methods (Kruskal-Wallis test and feature selection via concave minimization) and finally, SVM structure with high accuracy. The overall accuracy of $89 \%$ during the Beta2 frequency band is obtained effectively using a set of discriminative features using the proposed hierarchical method. 


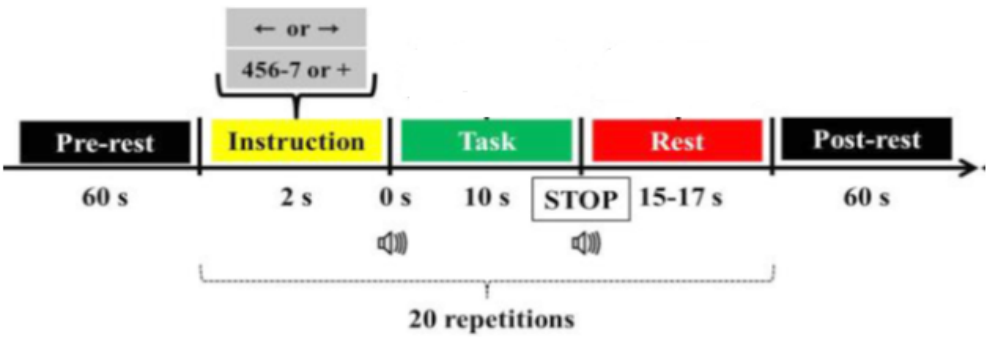

Figureure 1. Schematic sequence diagram of the experimental paradigm

NEUR SCIENCE

In investigating the causality and information flow in the brain for the mental arithmetic task, we had several options such as Dynamic Causal Modeling (DCM) and Granger causality tool based on structural equation modeling to extract effective connectivity. Granger causality is more straightforward in computation than DCM since it needs to compute autoregressive models instead of fitting statistical distributions using the expectationmaximization algorithm to construct DCM. We used SIFT toolbox to extract the GC measures. We tested all the measures and only reported the best measures (in the sense of classification performance). Results of GPDC features in the present study have the maximum accu- racy in differentiating mental arithmetic task vs. resting state. In many neurophysiological applications, localizing activity sources is more critical than sinks. Therefore, using connectivity measures like GPDC, proposed to indicate the sources, might be more relevant (Baccalá, Sameshima, \& Takahashi, 2007). Figure 4 shows strong sources (AFP1 and AFP2) in the frontal area during the mental arithmetic tasks compared to the resting state.

This study tested five widely used feature selection algorithms after the Kruskal-Wallis test. Our results observed wrapper methods that embedded feature selection methods like concave minimization and SVM yielded better clas-

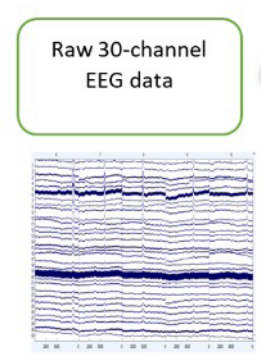

(a)
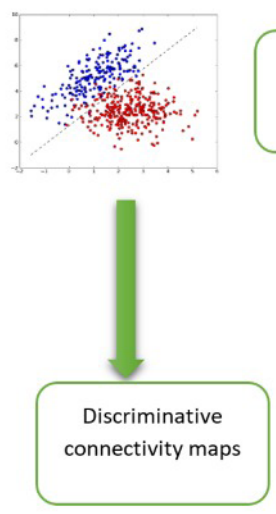

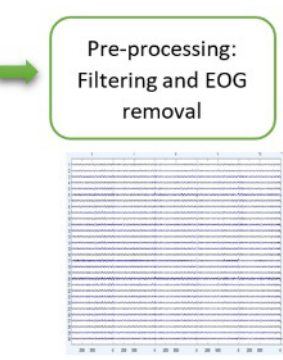

(b)

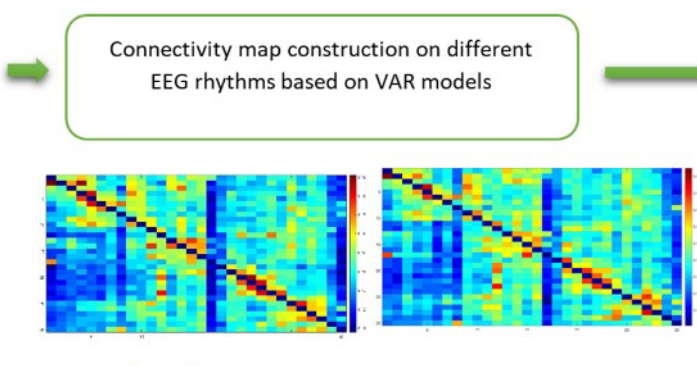

Mental Artihmetic

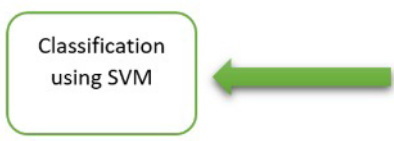

(e)

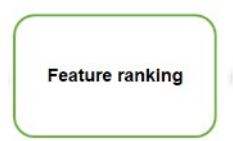

(d)

Figureure 2. The process of the proposed system

NEUR \$SCIENCE

A: Raw EEG data; B: Preprocessing; C: Construction of effective connectivity matrix; D: The statistical significance of the extracted connectivity features between mental arithmetic and baseline tasks using the Kruskal-Wallis test; E: Feature selection and ranking using five feature selection methods; F: Classification using SVM; G: Discriminative connectivity maps. 

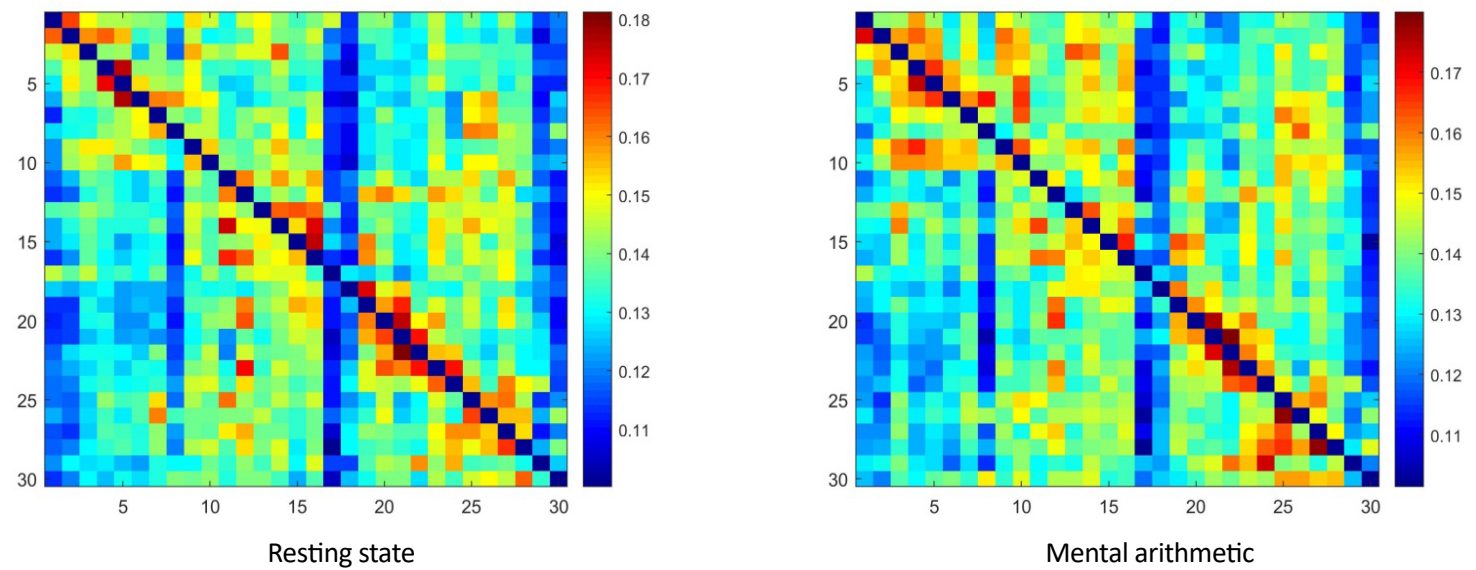

NEUR SCIENCE

Figure 3. Raw $900(30 \times 30)$ GPDC connectivity features for Beta2 frequency band over all participants for mental arithmetic task vs. resting state

A higher absolute value of connectivity feature shows with warm colors. Thirty electrodes are as follow: 1=F7, 2=AFF5h, 3=F3, 4=AFp1, 5=AFp2, 6=AFF6h, 7=F4, 8=F8, 9=AFF1h, 10=AFF2h, 11=Cz, 12=Pz, 13=FCC5h, 14=FCC3h, 15=CCP5h, 16=CCP3h, 17=T7, 18=P7, 19=P3, 20=PPO1h, 21=POO1, 22=POO2, 23=PPO2h, 24=P4, 25=FCC4h, 26=FCC6h, 27=CCP4h, 28=CCP6h, 29=P8, $30=\mathrm{T} 8$.

sification results. In this method, the feature selection part is combined with the training of an SVM as the classifier and cannot be separated. Moreover, embedded methods have better performance for feature selection since they use a classifier during the feature selection phase (Bradley, \& Mangasarian 1998). Other methods, such as filter methods (e.g., Fisher score, MI, \& mRMR), only consider the relevance of features with a dependent variable using statistical measures like correlation and ignoring the classifier. In contrast, wrapper methods like concave minimization and
SVM use a classifier, like SVM in feature selection. Previous studies have reported that theta, alpha, and beta bands are relatively sensitive to cognitive workload change (Lang et al., 2012; Bertsekas, 1995). Moreover, previous studies have indicated that when a participant is performing the mental arithmetic task, specific frequency components of EEG in frequency bands of 7-30 as a neural oscillation of brain electrophysiological activity are (de)synchronized, and best results of mental recognition are obtained (Harmony et al., 1999; Rebsamen et al., 2011; Wang, \& Sourina,

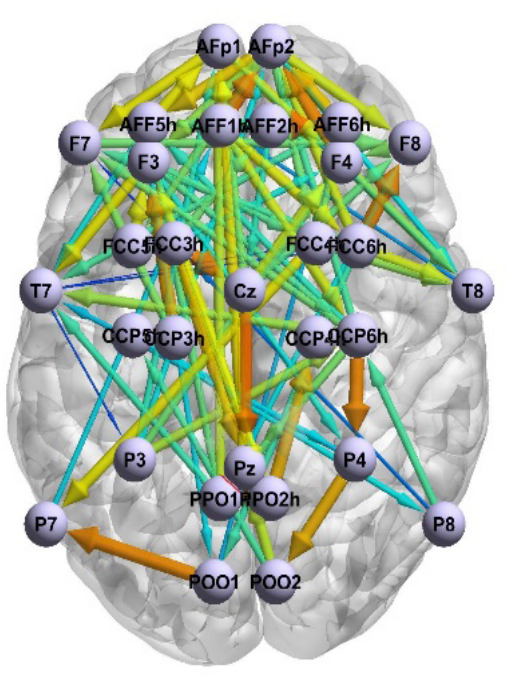

Resting state

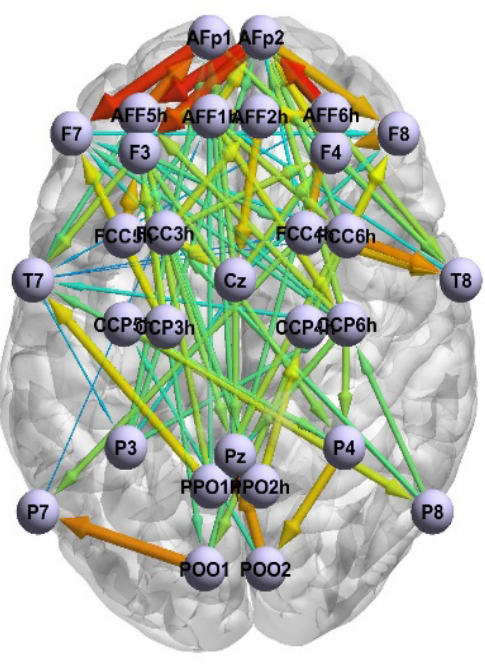

Mental arithmetic

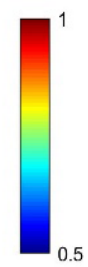

NEUR SCIENCE

Figure 4. The best GPDC connectivity features were obtained from feature selection via concave minimization for Beta2 frequency band over all participants for mental arithmetic task vs. resting state

A higher absolute value of connectivity feature shows with warm colors. The arrows represent directional connectivity. 
Table 1. Classification accuracy

\begin{tabular}{|c|c|c|c|c|c|c|}
\hline & & \multicolumn{5}{|c|}{ Mean \pm SD } \\
\hline \multicolumn{2}{|c|}{ GC Methods } & \multicolumn{5}{|c|}{ Feature Selection Methods } \\
\hline & & RFE & Fisher & MI & mRMR & Concave Minimization \\
\hline \multirow{3}{*}{ Theta band } & DTF & $50.2 \pm 2.94$ & $63.1 \pm 5.30$ & $56.3 \pm 2.10$ & $60.7 \pm 4.59$ & $67.8 \pm 3.82$ \\
\hline & dDTF & $54.1 \pm 2.75$ & $65.3 \pm 5.60$ & $58.2 \pm 3.39$ & $68.2 \pm 5.54$ & $71.2 \pm 4.42$ \\
\hline & GPDC & $58.6 \pm 4.75$ & $71.2 \pm 6.75$ & $60.5 \pm 5.94$ & $72.1 \pm 6.70$ & $77.5 \pm 6.39$ \\
\hline \multirow{3}{*}{ alpha band } & DTF & $55.1 \pm 1.93$ & $64.1 \pm 4.58$ & $58.3 \pm 3.07$ & $62.1 \pm 5.35$ & $70.2 \pm 5.20$ \\
\hline & dDTF & $58.1 \pm 1.19$ & $70.3 \pm 4.94$ & $60.2 \pm 2.67$ & $72.2 \pm 6.65$ & $74.1 \pm 6.71$ \\
\hline & GPDC & $59.3 \pm 2.74$ & $74.4 \pm 5.76$ & $65.5 \pm 4.86$ & $76.8 \pm 6.70$ & $82.3 \pm 6.79$ \\
\hline \multirow{3}{*}{ Beta1 band } & DTF & $56.2 \pm 3.42$ & $65.2 \pm 4.96$ & $57.3 \pm 4.58$ & $65.1 \pm 5.25$ & $72.5 \pm 6.53$ \\
\hline & dDTF & $60.2 \pm 3.11$ & $72.1 \pm 5.28$ & $62.1 \pm 3.48$ & $74.1 \pm 4.09$ & $76.1 \pm 6.71$ \\
\hline & GPDC & $61.3 \pm 4.52$ & $78.2 \pm 4.75$ & $67.4 \pm 2.38$ & $79.2 \pm 5.56$ & $85.7 \pm 6.82$ \\
\hline \multirow{3}{*}{ Beta2 band } & DTF & $56.8 \pm 3.83$ & $72.1 \pm 5.88$ & $62.8 \pm 2.46$ & $75.1 \pm 5.81$ & $78.2 \pm 5.23$ \\
\hline & dDTF & $62.1 \pm 2.44$ & $74.7 \pm 5.25$ & $67.3 \pm 4.31$ & $78.2 \pm 4.09$ & $85.2 \pm 6.39$ \\
\hline & GPDC & $65.3 \pm 3.31$ & $80.2 \pm 6.23$ & $70.2 \pm 5.70$ & $82.6 \pm 6.50$ & $89.2 \pm 4.89$ \\
\hline \multirow{3}{*}{ Beta3 band } & DTF & $53.1 \pm 1.78$ & $63.3 \pm 5.27$ & $57.2 \pm 3.69$ & $68.2 \pm 4.82$ & $69.2 \pm 4.57$ \\
\hline & dDTF & $54.1 \pm 2.00$ & $65.3 \pm 5.30$ & $59.3 \pm 2.35$ & $70.2 \pm 5.56$ & $72.3 \pm 5.56$ \\
\hline & GPDC & $58.9 \pm 3.84$ & $71.4 \pm 6.53$ & $63.2 \pm 5.45$ & $74.7 \pm 5.23$ & $80.6 \pm 6.42$ \\
\hline \multirow{3}{*}{$\begin{array}{l}\text { Gamma- } \\
\text { band }\end{array}$} & DTF & $52.8 \pm 2.66$ & $60.1 \pm 5.74$ & $55.3 \pm 3.84$ & $61.7 \pm 3.01$ & $66.2 \pm 4.57$ \\
\hline & dDTF & $53.1 \pm 3.97$ & $64.8 \pm 4.54$ & $59.3 \pm 4.02$ & $65.2 \pm 4.35$ & $70.8 \pm 5.46$ \\
\hline & GPDC & $57.6 \pm 3.71$ & $68.5 \pm 5.12$ & $62.5 \pm 4.53$ & $70.2 \pm 5.21$ & $75.1 \pm 6.69$ \\
\hline
\end{tabular}

NEUR SCIENCE

2013; Lang et al., 2012; Smith, Gevins, Brown, Karnik, \& Du, 2001). This study on mental arithmetic vs. resting state pattern discrimination is per previous results. The results of the Delta band were not promising and were below 50\%; therefore, it was omitted in the report. The best results of mental arithmetic recognition and significant changes in the effective connectivity are mainly from the alpha and beta bands, especially in the beta 2 band.

Our network analysis revealed during the mental arithmetic task that EEG generally shows high separability around frontal and prefrontal areas (Figure 4, especially AFP1 and AFP2). The effective connectivity varied and increased mostly around these areas' frequency bands. In other words, most discriminative beta-band effective connections are exhibited around the frontal and prefron- tal regions. Neuropsychological findings demonstrated that cognitive workload is done in the prefrontal cortex (Fernández et al., 1995; Brass, Ullsperger, Knoesche, von Cramon, \& Phillips, 2005). So our results might serve as evidence for the correlation of the frontal lobe with the cognitive workload. According to this result, prefrontal and frontal regions contribute to the visual recognition of numbers and are vital for mental calculation. Our results have highlighted the interaction of the prefrontal with central, temporal, and parietal cortices. Time-lag and causal effects between prefrontal and central, temporal, parietal cortices explain how mental calculation can be matched to enable a comprehensible cognitive function.

The results of directional connectivity metrics (GPDC features) in multichannel EEG in the present study infer that infor- 
mation flow from different parts of the brain to the other plays a vital role in the differentiation of mental arithmetic tasks vs. resting state. As shown in Figure 4, differential patterns of the best selected GPDC connectivity features obtained from KruskalWallis test and feature selection via concave minimization for Beta2 frequency are around frontal and prefrontal areas. Also, the accuracies attained with our method $(89 \%)$ outperform the other methods that use EEG features from individual channels (80\%) Shin et al. (2017) in the same database. To mention a limitation of our study, we believe that the performance of a multi-modal system based on EEG and Near-Infrared Spectroscopy (NIRS), compared with a single modality, might improve accuracy. Finally, the performance of the system of discrimination of mental arithmetic tasks might be improving.

\section{Conclusion}

This study addresses a new automated system based on effective connectivity quantified with the GPDC method and a hierarchical machine learning structure methods for discrimination of mental arithmetic task vs. resting state from EEG signals with an accuracy of $89 \%$ on the 29 participants within beta 2 frequency band. Results indicate that exploring causal dependencies between participants' brain regions using directed information flow plays an important role and has potential discriminative value.

\section{Ethical Considerations}

\section{Compliance with ethical guidelines}

All procedures performed in studies involving human participants were in accordance with the ethical standards of the institutional research committee with the 1964 Helsinki declaration. This study was done according to the Ethics Committee of the Technical University of Berlin (SH_01_20150330).

Funding

The article was supported by Medicine Shahid Beheshti University of Medical Sciences.

Authors' contributions

All authors equally contributed to preparing this article.

Conflict of interest

The authors declared no conflict of interest.

\section{Refrences}

Afshani, F., Shalbaf, A., Shalbaf, R., \& Sleigh, J. (2019). Frontaltemporal functional connectivity of EEG signal by standardized permutation mutual information during anesthesia. Cognitive Neurodynamics, 13(6), 531-40. [DOI:10.1007/s11571019-09553-w] [PMID] [PMCID]
Akbarian, B., \& Erfanian, A. (2018). Automatic seizure detection based on nonlinear dynamical analysis of eeg signals and mutual information. Basic and Clinical Neuroscience, 9(4), 227-40. [DOI:10.32598/bcn.9.4.227] [PMID] [PMCID]

Astolfi, L., Cincotti, F., Mattia, D., Marciani, M. G., Baccala, L. A., \& de Vico Fallani, F., et al. (2007). Comparison of different cortical connectivity estimators for high-resolution EEG recordings. Human Brain Mapping, 28(2), 143-57. [DOI:10.1002/hbm.20263] [PMID] [PMCID]

Baccalá, L. A., \& Sameshima, K. (2001). Partial directed coherence: A new concept in neural structure determination. Biological $\mathrm{Cy}$ bernetics, 84, 463-74. [DOI:10.1007/PL00007990] [PMID]

Baccalá, L. A., Sameshima, K., \& Takahashi, D. Y. (2007). Generalized partial directed coherence in digital signal processing. International Conference on Digital Signal Processing,163-66. [DOI:10.1109/ICDSP.2007.4288544]

Bertsekas,D.P.(2016). Nonlinearprogramming. Massacusetts: Athenas Scientific, Belmont. https://www.google.com/books/edition/ Nonlinear_Programming/rC1EEAAAQBAJ?hl=en\&gbpv $=0$

Bradley, P. \& Mangasarian, O. (1998). Feature selection via concave minimization and support vector machines. Paper present at The $15^{\text {th }}$ International Conference (ICML '98). Burlington, United States, 24 July 1998. http://citeseerx.ist.psu.edu/viewdoc/ versions?doi=10.1.1.52.5153

Brass, M., Ullsperger, M., Knoesche, T. R., von Cramon, D. Y., \& Phillips, N. A. (2005). Who comes first? The role of the prefrontal and parietal cortex in cognitive control. Journal of Cognitive Neuroscience, 17(9), 1367-1375. [DOI:10.1162/0898929054985400] [PMID]

Delorme, A., \& Makeig, S. (2004). EEGLAB: An open source toolbox for analysis of single-trial EEG dynamics including independent component analysis. Journal of Neuroscience Methods, 134(1), 9-21. [DOI:10.1016/j.jneumeth.2003.10.009] [PMID]

Dimitrakopoulos, G. N., Kakkos, I., Dai, Z., Lim, J., deSouza, J. J., \& Bezerianos, A., et al. (2017) Task-independent mental workload classification based upon common multiband EEG cortical connectivity. IEEE Transactions on Neural Systems and Rehabilitation Engineering, 25 (11), 1940-9. [DOI:10.1109/TNSRE.2017.2701002] [PMID]

Dimitriadis, S. I., Sun, Y., Kwok, K., Laskaris, N. A., \& Bezerianos, A. (2013). A tensorial approach to access cognitive workload related to mental arithmetic from EEG functional connectivity estimates. Annual International Conference of the IEEE Engineering in Medicine and Biology Society, 2013, 2940-3. [DOI:10.1109/ EMBC.2013.6610156] [PMID]

Dimitriadis, S. I., Sun, Y., Thakor, N. V., \& Bezerianos, A. (2016) Causal interactions between frontal-parietal-occipital predict performance on a mental arithmetic task. Frontiers in Human Neuroscience, 10, 454. [DOI:10.3389/fnhum.2016.00454] [PMID] [PMCID]

Ding, C. \& Peng, H. (2005). Minimum redundancy feature selection from microarray gene expression data. Journal of Bioinformatics and Computational Biology, 3(2), 185-205. [DOI:10.1142/ S0219720005001004] [PMID]

Fernández, T., Harmony, T., Rodríguez, M., Bernal, J., Silva, J., \& Reyes, A., et al. (1995). Eeg activation patterns during the performance of tasks involving different components of mental calculation. Electroencephalography and Clinical Neurophysiology, 94(3), 175-82. [DOI:10.1016/0013-4694(94)00262-J] [PMID] 
Geweke, J. F. (1984). Measures of conditional linear dependence and feedback between time series. Journal of the American Statistical Association, 79(388), 907-15. [DOI:10.1080/01621459.1984.1047 7110]

Granger, C. W. J. (1969). Investigating causal relations by econometric models and cross-spectral methods. The Econometric Society, 37, 424-38. [DOI:10.2307/1912791]

Gu, Q., Li, Z., \& Han, J. (2011). Generalized fisher score for feature selection. Paper present at The 27th International Conference on Uncertainty in Artificial Intelligence. Arlington, Virginia, United States, 14 July 2011. [DOI:10.5555/3020548.3020580]

Guyon, I., Weston, J., Barnhill, S., \& Vapnik, V. (2002) Gene selection for cancer classification using support vector machines. Machine Learning, 46, 389-422. [DOI:10.1023/A:1012487302797]

Harmony, T., Fernández, T., Silva, J., Bosch, J., Valdés, P., \& Fernández-Bouzas, A., et al. (1999). Do specific EEG frequencies indicate different processes during mental calculation? Neuroscience letters, 266(1), 25-8. [DOI:10.1016/S0304-3940(99)00244-X] [PMID]

Kaminski, M., Ding, M., Truccolo, W. A., \& Bressler, S. L. (2001) Evaluating causal relations in neural systems: Granger causality, directed transfer function and Statistical assessment of significance. Biological Cybernetics, 85(2), 145-57. [DOI:10.1007/ s004220000235] [PMID]

Korzeniewska, A., Mańczak, M., Kamiński, M., Blinowska, K. J., \& Kasicki, S. (2003). Determination of information flow direction among brain structures by a modified directed transfer function (dDTF) method. Journal of Neuroscience Methods, 125(1-2), 195-207. [DOI:10.1016/S0165-0270(03)00052-9] [PMID]

Lang, E. W., Tome, A., Keck, I. R., Gorriz, J., \& Puntonet, C. (2012) Brain connectivity analysis: A short survey. Computational Intelligence and Neuroscience, 2012, 412512. [DOI:10.1155/2012/412512] [PMID] [PMCID]

Liang, N. Y., Saratchandran, P., Huang, G. B., \& Sundararajan, N. (2006). Classification of mental tasks from EEG signals using extreme learning machine. International Journal of Neural Systems, 16(1), 29-38. [DOI:10.1142/S0129065706000482] [PMID]

Lubar, J. F., Swartwood, M. O., Swartwood, J. N., \& O'Donnell, P. H. (1995). Evaluation of the effectiveness of EEG neurofeedback training for ADHD in a clinical setting as measured by changes in T.O.V.A. scores, behavioral ratings, and WISC-R performance. Biofeedback and Self-Regulation, 20(1), 83-99. [DOI:10.1007/ BF01712768] [PMID]

McFarland, D. J., \& Wolpaw, J. R. (2008). Brain-computer interface operation of robotic and prosthetic devices. Computer, 41(10), 52-6. [DOI:10.1109/MC.2008.409]

Miller, E. K., \& Cohen, J. D. (2001). An integrative theory of prefrontal cortex function. Annual Review of Neuroscience, 24, 167-202. [DOI:10.1146/annurev.neuro.24.1.167] [PMID]

Mohseni, H. R., Maghsoudi, A., \& Shamsollahi, M. B. (2006). Seizure detection in EEG signals: A comparison of different approaches. International Conference of the IEEE Engineering in Medicine and Biology Society, 6724-7. [DOI:10.1109/IEMBS.2006.260931] [PMID]

Mullen, T., Delorme, A., Kothe, C., \& Makeig, S. (2010). An electrophysiological information flow toolbox for EEGLAB. Biological Cybernetics, 83, 35-45. https://sccn.ucsd.edu/ scott/pdf/Mullen10_SIFT_SfN10.pdf
Neghabi, M., Marateb, H. R., \& Mahnam, A. (2019). Comparing steady-state visually evoked potentials frequency estimation methods in brain-computer interface with the minimum number of eeg channels. Basic and Clinical Neuroscience, 10(3), 245-56. [DOI:10.32598/bcn.9.10.200] [PMID] [PMCID]

Peng, H., Long, F., \& Ding, C. (2005). Feature selection based on mutual information: Criteria of max-dependency, maxrelevance, and minredundancy. IEEE Transactions on Pattern Analysis and Machine Intelligence, 27(8), 1226-38. [DOI:10.1109/ TPAMI.2005.159] [PMID]

Rebsamen, B., Kwok, K., \& Penney, T. B. (2011). Evaluation of cognitive workload from EEG during a mental arithmetic task. Proceedings of the Human Factors and Ergonomics Society Annual Meeting, 55(1), 1342-45. [DOI:10.1177/1071181311551279]

Seth, A. K. (2010). A matlab toolbox for granger causality connectivity analysis. Journal of Neuroscience Methods, 186(2), 26273. [DOI:10.1016/j.jneumeth.2009.11.020] [PMID]

Shalbaf, A., Shalbaf, R., Saffar, M., \& Sleigh, J. (2020) Monitoring the level of hypnosis using a hierarchical SVM system. Journal of Clinical Monitoring and Computing, 34(2), 331-8. [DOI:10.1007/s10877-019-00311-1] [PMID]

Shin, J., von Luhmann, A., Blankertz, B., Kim, D. W., Jeong, J., \& Hwang, H. J., et al. (2017). Open access dataset for eeg+nirs single-trial classification. IEEE Transactions on Neural Systems and Rehabilitation Engineering, 25(10), 1735-45. [DOI:10.1109/ TNSRE.2016.2628057] [PMID]

Smith, M. E., Gevins, A., Brown, H., Karnik, A., \& Du, R. (2001). Monitoring task loading with multivariate eeg measures during complex forms of human-computer interaction. Human Factors, 43(3), 366-80. [DOI:10.1518/001872001775898287] [PMID]

Spurrier, J. D. (2003). On the null distribution of the kruskalwallis statistic. Journal of Nonparametric Statistics, 15(6), 685-91. [DOI:10.1080/10485250310001634719]

Tajar, J., \& Sharifi, S. (2011). Dyscalculia: Learning disabilites in mathematice and treatment with teaching remedial method Iranian children 6 years old. Australian Journal of Basic and Applied Sciences, 5(9), 891-6. http://www.ajbasweb.com/old/ ajbas/2011/September-2011/891-896.pdf

Thompson, L., Thompson, M., \& Reid, A. (2010). Neurofeedback outcomes in clients with asperger's syndrome. Applied Psychophysiology and Biofeedback, 35(1), 63-81. [DOI:10.1007/s10484009-9120-3] [PMID]

Wang, Q., \& Sourina, O. (2013). Real-time mental arithmetic task recognition from eeg signals. IEEE Transactions on Neural Systems and Rehabilitation Engineering, 21(2), 225-32. [DOI:10.1109/TNSRE.2012.2236576] [PMID]

Wolpaw, J. R., Birbaumer, N., McFarland, D. J., Pfurtscheller, G. \& Vaughan, T. M. (2002) Brain-computer interfaces for communication and control. Clinical Neurophysiology, 113(6), 767-91. [DOI:10.1016/S1388-2457(02)00057-3] [PMID]

Zaffalon, M., \& Hutter, M. (2002) Robust feature selection using distributions of mutual information. Paper present at The $18^{\text {th }}$ International Conference on Uncertainty in Artificial Intelligence. San Francisco, CA, United States, 1 August 2002. [DOI:10.5555/2073876.2073945] 
This Page Intentionally Left Blank 